\title{
School Leaders' Experiences on Dealing with Students Exposed to Domestic Violence
}

\author{
Emily Berger $^{1}$ (D) $\cdot$ Natalie Chionh ${ }^{1} \cdot$ Amy Miko $^{1}$
}

Accepted: 20 August 2021 / Published online: 24 August 2021

(c) The Author(s), under exclusive licence to Springer Science+Business Media, LLC, part of Springer Nature 2021

\begin{abstract}
School leaders' experiences and perceptions of students' domestic violence experiences and trauma-informed school interventions have been overlooked. This semi-structured qualitative study aimed to investigate three areas regarding school leaders' perceptions regarding domestic violence-exposed students: 1) the experiences of school leaders in dealing with these students; 2) the barriers and strengths associated with managing these students; and 3) school leaders' recommendations for overcoming any barriers related to this issue in schools. Interviews conducted with 15 school leaders were analysed using thematic analysis. School leaders were found to be instrumental in influencing school culture and support systems surrounding students' exposure to domestic violence. However, school leaders provided mixed reports regarding the adequacy of resources for domestic violence-exposed students. Recommendations suggested by school leaders included more domestic violence specific training for school staff and training delivery methods that were more sensitive for staff who have personal experiences with domestic violence. School leaders expressed great concerns for teachers, particularly less experienced teachers, regarding their knowledge and skills to assist students who disclose family violence.
\end{abstract}

Keyword Domestic violence; principals; schools; trauma-informed; students

\section{The Nature and Extent of Domestic Violence}

Domestic violence (DV) is defined as harm inflicted upon others of intimate or familiar relationships, past or present (Australian Government, 2011). Also known as family violence or intimate partner violence, DV can include physical, emotional, sexual or psychological abuse between spouses or former spouses, or between family members, such as violence inflicted by a parent against a child (Department of Families, Housing, Community Services and Indigenous Affairs [FaHCSIA] \& Council of Australian Governments [COAG], 2011). DV is a highly complex issue which is influenced by a range of systemic and interpersonal variables, and DV affects families worldwide (Garcia-Moreno et al., 2006; Yates, 2020). Gender is regarded as an important factor when considering DV. Research consistently suggests that DV is a gendered crime, with the United Nations (United Nations Population Fund, 2000) estimating between

Emily Berger

emily.berger@monash.edu

1 Faculty of Education, Monash University, 19 Ancora Imparo Way, Clayton, VIC 3800, Australia
20 and 50 percent of females have experienced some form of violence perpetrated by a partner or family member (Kuskoff and Parsell, 2019). Although the connection between gender and DV is contentious, females are more likely to be victimised by male partner or husband, compared to males who are victimised by their female partner (Boxall et al., 2020; Kuskoff and Parsell, 2019; Yates, 2020). The Victorian Royal Commission into Family Violence reported "family violence disproportionately affects women and children, and the majority of perpetrators are men" (Neave et al., 2016, p. 18). While males and females experience DV, females often engage in aggressive behaviours as a defence against a violent partner (Boxall et al., 2020), while males are more likely to engage in controlling behaviour towards their female spouse (Garcia-Moreno et al., 2006; Yates, 2020).

Research has also considered LGBTQI couples in the context of DV, however, a larger portion of DV studies refer to genders as 'female' and 'male' without specifying whether participants identify as cis or transgender. A study with lesbian, gay, bisexual, trans, queer and intersex (LGBTQI) couples found inter-partner violence is as common between these couples as cisgender, heterosexual couples (Campo \& Tayton, 2015). Another study reported DV 
is more prevalent within LGBTQI young adult couples when compared to cisgender, heterosexual relationships, with female-identifying LGBTQI individuals more at risk of DV than male-identifying LGBTQI individuals (Reuter et al., 2017). Another study by Pitts and colleagues (2006) found approximately $28 \%$ of male-identifying participants were in a relationship with an abusive partner, compared to $41 \%$ of female-identifying participants. Evidently, DV affects adults within both cisgender, heterosexual and LGBTQI communities. The consequences of DV extend beyond adults to any children in their care.

\section{The Impact of Domestic Violence on Children}

Children are victimised by DV through witnessing the violence between parents or caregivers, or through direct involvement in the violence, such as protection of the victimised parent or younger siblings (FaHCSIA \& COAG, 2011). Further, other childhood stressors are linked to violence within the home, such as parent mental illness and psychological distress, parental substance abuse, parent separation and divorce, and stigma and social isolation attributed to exposure to DV (Levendosky \& Graham-Bermann, 2001; Levendosky et al., 2006; Whitaker et al., 2006). The culmination of stressors results in children's frequent worry about the safety of family members and stability of their family unit (Buckley et al., 2007). These children may experience reduced self-esteem, heightened anxiety and a sense of being 'different,' affecting their functioning across a range of settings (Buckley et al., 2007). In particular, the emotional, physical and cognitive impacts of DV on children, such as heightened stress, difficulty concentrating, difficulty regulating emotions and potential for physical injury, influence a child's schooling experience (Lloyd, 2018). These children experience delayed learning and increased social and behavioural problems, which require a response from school staff (Lloyd, 2018). Thus, it is imperative educators are equipped to understand and respond to disclosures and the impacts of DV on children.

\section{Teachers' Experiences of Handling Domestic Violence}

Students impacted by DV may engage in a range of challenging behaviours at school. The concern is that these challenging behaviours may be misinterpreted by educators as misbehaviour, and punished, which may perpetuate the stress cycle for students (Iacona \& Johnson, 2018). Teachers have reported their lack of knowledge and skills to respond to students exposed to DV (Davies \& Berger, 2019). Davies and Berger (2019) found teachers require training, school policies and procedures to manage the emotional burden of families experiencing DV. Further, teachers required formalised frameworks to inform their response to DV-exposed students (Davies \& Berger, 2019). Years of experience working in schools and as a teacher is associated with greater confidence and perceived skills for identifying, managing and reporting instances of student DV and trauma exposure (Alisic, 2012; Davies \& Berger, 2019; Mckee \& Dillenburger, 2009; Smith, 2010). Ultimately, students spend a considerable amount of time at school and the capacity of teachers to respond to DV and maintain positive, trusting and low-conflict relationships with students can play an important protective role for students (Vaughan-Jensen et al., 2020).

\section{The Role of School Leaders}

For this study, a school leader is defined as a school staff member in a managerial role who oversees and guides the operations of a school, such as school principals, assistant principals and school/year level coordinators. School leaders have a central role in defining the mission statement and values of their school and allocation of resources and time for their teachers to attend professional learning activities (Benoliel, 2017; Drysdale \& Gurr, 2011). Thus, school leaders inform the resolve and skills of teachers in response to students' exposure to DV (Howard et al., 2018). Although school leaders may not have ample direct interaction with students, Moore (2009) reported clear flow-on effects between empathetic school leadership, teachers' classroom and behaviour management practices, implementation of school support programs for students and teachers' effective emotional support of students. In the study by Davies and Berger (2019), teachers identified reliance on the school principal to manage instances of student exposure to DV. One teacher reported on their experiences of student DV exposure, "We feel pretty comfortable in the way that we support each other, because we know that our principal, our leadership, certainly has our back" (Davies \& Berger, 2019, p. 101). However, teachers do not always experience support from their school principal in response to students' DV exposure, with one participant reporting, "It was rubbish...the assistant principal didn't have the right skills basically" (Davies \& Berger, 2019, p. 101). The limited literature on school leaders' role in dealing with students exposed to DV suggests more research is needed on the experiences and potential challenges of school leaders when responding to students who have experienced DV, and in supporting the teachers who support these students. 


\section{Mandatory Reporting}

Mandatory reporting laws in Australia are also important in dictating the response of teachers, school leaders and other school staff concerning students' exposure to DV and other forms of violence and neglect. Mandatory reporting laws vary between Australian states (Australian Institute of Family Studies [AIFS], 2020). Victorian law states that school staff who form a reasonable belief a student has been exposed to violence or neglect (and the parents are unable or unwilling to protect the student), then a report must be made by the staff member to child protection services (State Government of Victoria, 2019). However, research has found that while the introduction of these laws increased reporting by school staff in relation to issues of child abuse or neglect, many teachers remain confused and ill-equipped to manage their mandatory reporting responsibility (Falkiner et al., 2017; Mathews et al., 2016).

In light of the above, the aim of this study was to explore the experiences of school leaders when responding to students exposed to DV. A qualitative approach was chosen for this study to replicate earlier research on the experiences and perceptions of teachers regarding student exposure to DV and trauma (e.g. Davies \& Berger, 2019). Outcomes of this study are anticipated to provide recommendations for improved professional development, school policy and collaboration between school and community services regarding students' experiences of DV. To explore this topic, three research questions were formulated:

1. What are the experiences of school leaders in relation to dealing with students exposed to DV, if any?

2. What barriers do school leaders perceive in dealing with students exposed to DV, if any?

3. What do school leaders recommend to overcome the barriers in response to students exposed to DV, if anything?

\section{Method}

\section{Participants}

Participants were school leaders situated in Victoria, Australia. The gender, role and school levels managed by participants are illustrated in Table 1. Participants were aged between 25 to 60 years $\left(M_{\text {age }}=45.13, S D=11.03\right)$. The participants had a range of four to 32 years working in schools $(M=20.37, S D=9.34)$. All participants identified as either male or female. No participants identified as a gender other than male or female. A total of 15
Table 1 Participant's Gender, Role and School Level Managed

\begin{tabular}{lllll}
\hline & \multicolumn{2}{l}{ School Leader Roles } & \\
\cline { 2 - 4 } & $\begin{array}{l}\text { School } \\
\text { Principal }\end{array}$ & $\begin{array}{l}\text { Assistant } \\
\text { Principal }\end{array}$ & $\begin{array}{l}\text { Year-Level } \\
\text { Coordinator }\end{array}$ & Total \\
\hline Gender & & & & \\
Male & 2 & - & 1 & 3 \\
Female & 5 & 3 & 4 & 12 \\
Total & 7 & 3 & 5 & 15 \\
School Level & & & & \\
Primary & 4 & 1 & - & 5 \\
Secondary & 1 & 2 & 5 & 1 \\
Combined & 1 & - & - & 1 \\
Special Ed & 1 & - & - & 15 \\
Total & 7 & 3 & 5 & \\
\hline
\end{tabular}

Note. Primary schools are catered to students aged 5 to 12 years old. Secondary schools are catered to students aged 12 to 18 years old. Combined refers to school leaders working in primary and secondary schools. Special Ed refers to special education settings for students with additional learning, behavioural and/or emotional challenges

participants agreed to participate in an interview following completion of a survey as part of the larger study into school staff experiences with traumatised students. The current sample were all school leaders who agreed to participate in an interview after they completed the survey.

\section{Materials}

A semi-structured interview schedule was developed based on a constructivist theoretical approach (see section on design and analysis for more information). The interview schedule was created by the researchers and included questions regarding the experiences, school protocols, and support and training needs of school leaders and school staff in the area of DV. To address the research questions, a list of open-ended questions was used during the interviews with school leaders (Table 2). These questions were followed by varying probing questions asked of school leaders based on their responses to the central questions. Participants were provided with the following definition of DV before the interview commenced: Student exposure to domestic violence may be defined as any experiences of students were they have heard, seen, become directly involved in, such as attempting to intervene, or experienced the aftermath of physical or sexual violence between their caregivers or significant others.

\section{Procedure}

Approval was obtained from the Monash University Human Research Ethics Committee, the Victorian Department of Education, and the Catholic Education Office of Melbourne. 
Table 2 Semi-Structured Interview Questions

\begin{abstract}
Questions
What is your experience with regard to students and domestic violence, if any?

What are the most common types of domestic violence that students are exposed to, if any?

Does your school have a protocol with regard to students and domestic violence?

How do you talk about the topic of students and domestic violence with colleagues, if at all?

What can you tell me about your experience of parents or families who may have experienced domestic violence, if any?

Have you ever undertaken training in this area?
\end{abstract}

All school principals in Victoria were approached via email and asked to consent to the study. Principals who agreed to the study $(N=83)$ then emailed the project information sheet to other school leaders at their school. Within the information sheet, the purpose of the study was outlined and the numbers of mental health services were included. School leaders interested in participating in an interview contacted the researchers. The interviews with school leaders was one component of a larger study that included interviews and questionnaires with school leaders, teachers and school mental health staff. Verbal consent was obtained from school leaders prior to their participation in a telephone interview.

Members of the research team conducted the interviews. The researchers are either provisionally or fully registered psychologists with the Psychology Board of Australia and have several years of qualitative research experience. Demographic questions were asked of all participants verbally prior to commencing the interview, such as age, self-identified gender and years of experience working in schools. Audio devices were used to record the interviews with school leaders. A professional transcription company transcribed the recorded interviews verbatim. No problems were noted by the company regarding the veracity or intelligibility of audio files. School leaders were emailed their transcript and invited to provide any additional information in a process known as member checking. No school leader opted to provide additional information and all data were retained for analysis.

\section{Design and Analysis}

Thematic analysis was used to analyse data and to identify themes and patterns of school leaders' experiences in dealing with students exposed to DV. As the study was exploratory in nature and not guided by any predetermined expectations about the content of the interviews, thematic analysis was chosen as the qualitative analysis technique to analyse the data (Braun \& Clarke, 2006). A constructivist perspective guided the qualitative methodology and analysis for this paper. A constructivist perspective can be used when there is limited understanding about a topic and when knowledge on a topic has been constructed based on the prior experiences of participants (Savin-Baden \& Howell Major, 2013). Both of these assumptions were believed to be true in relation to the topic of school leaders and their responses to student exposure to DV. The process of thematic analysis comprises six steps: (1) familiarisation of the researchers with the data; (2) identifying themes (ideas) in the data; (3) developing themes to reflect the data; (4) reviewing themes and creating subthemes of related ideas; (5) defining themes; and (6) writing up the themes (Braun \& Clarke, 2006). Familiarisation of the data consisted of the first two authors individually reading, re-reading and understanding the content of all of the transcripts. Both authors then individually collated the data into themes and subthemes. The authors then came together to review their themes and subthemes and to reach consensus on the final set of themes and subthemes. An independent researcher then analysed two transcripts for the purposes of double coding. This independent researcher was someone with qualitative training but not involved in collection or initial analysis of the data. Consultation then occurred between the independent researcher and the first two authors. No new themes were identified by the independent researcher that had not already been established by the authors.

\section{Results}

The thematic analysis conducted by the authors revealed five main themes (with the corresponding subtheme(s) included alongside each main theme; see Table 3). Quotes are provided with the themes to illustrate each theme and subtheme. Each quote is labelled with the corresponding participant's identified gender, age, years of experience in schools and role within the school to show the diversity of participants in the study.

\section{School Leaders' Experiences with Students' Exposed to DV}

Impact of DV on Students Participants reported that students exposed to DV present as withdrawn, have unpredictable outbursts or aggression, are unable to regulate their 
Table 3 Themes and Subthemes Generated from the Interviews and Thematic Analysis

\begin{tabular}{|c|c|}
\hline Themes & Subtheme(s) \\
\hline 1. School Leaders' Experiences with Students' Exposed to DV & $\begin{array}{l}\text { - Impact of DV on students } \\
\text { - Impact of DV on school leaders } \\
\text { - Gender of school leaders and relationship with students }\end{array}$ \\
\hline 2. Mixed levels of support and training for schools & $\begin{array}{l}\text { - Mixed levels of trauma-based and trauma-informed practices for handling DV } \\
\text { - Differing levels of support for schools to deal with parents and families } \\
\text { - Mixed levels of resources and training for school staff to support students }\end{array}$ \\
\hline 3. Levels of school support from government for DV victims & $\begin{array}{l}\text { - Funding for schools } \\
\text { - Support from external agencies }\end{array}$ \\
\hline 4. Role of school wellbeing officers & $\begin{array}{l}\text { - Reliance on school wellbeing officers for support } \\
\text { - School wellbeing departments' knowledge of mental health support }\end{array}$ \\
\hline
\end{tabular}

emotions, and appear hypervigilant and suffer from depression. Participants attributed students' behavioural problems to having poor role models within the home. Students' low academic performance was attributed by participants to students' hypervigilance regarding their DV situations and concerns about their mother's safety, and to their inability to regulate their emotions and to concentrate at school. However, participants reported that students felt that school was a safe place for them. Participants cited that they can struggle when students want more days at school rather than to be at home, as participants felt that these students actually needed extra days off from school due to poor coping in the school setting. This was exemplified by one participant who stated, “...if you've witnessed DV, you don't feel safe at home and so they're constantly, well they're either hyper-aroused where they're going a million miles a minute and they're ready to go from nought to a hundred really quickly, because they're just constantly looking for that threat" (Female, aged 36, 9 years, Principal). Similarly, another participant reported, "The other kids were often very reluctant to engage with him because they weren't sure what his response was going to be...when he turned violent he basically started overturning furniture and throwing things" (Male, aged 59, 32 years, Principal).

Many participants reported finding it hard to support students with the little information which they can gather about students' experiences of DV. Participants also reported a culture of secrecy regarding DV, where the topic of DV was avoided by adults and whereby students were taught not to say anything about the topic. In regards to having access to information, one participant stated, "It varies in degree and exposure and we are often not privy to it until we have outside agencies that contact us because it just - the way that the community is pretty tight lipped our students are taught not to say anything” (Female, aged 36, 15 years, Principal). Participants suggested that school staff found it hard to help students due to the lack of willingness of children to share their experiences.

Impact of DV on School Leaders Participants reported that dealing with students exposed to DV had emotional impacts on them. Participants felt that they were not well equipped to provide the type of mental health support required for these students and their parents. This lead to feelings of selfdoubt in their capacity to offer meaningful assistance. One participant stated, "I've got a really prickly family who I'm dealing with today, but you know at what cost, and now I question, do I have the skills that are required to actually be in this community?" (Female, aged 47, 20 years, Principal). Another participant reported not knowing how to support male parents who have experienced DV as most resources were aimed at females who have experienced DV.

While some participants provided informal debriefing to other school staff, other participants utilised a system whereby more experienced education staff members debriefed with less experienced staff. For example, one participant stated, “...we also talk about staff mentors, and we go about, I'm really lucky, I've got, there's 5 of us, and so there's only 12 teachers, so we're able to divvy up those teachers to who works well with who" (Female, aged 47, 20 years, Principal).

Participants also reported difficulties dealing with aggressive parents. In particular, participants experienced a dilemma between updating parents on their child's engagement or behaviour at school, while also protecting students from parental aggression, which occurs as a result of sharing negative updates on students with parents. One participant stated, "So it made it really difficult... because sometimes we were I guess a little bit reluctant to give feedback that we, if we knew there was going to be potential consequences at home...but at the same time we obviously needed to give feedback, because that's our responsibility as teachers" (Female, aged 36, 9 years, Principal). 
Gender of School Leaders and Relationship with Students One male participant reported unease in dealing with issues of abuse with students as he felt that male school staff were more likely to be distrusted compared to female staff. The participant stated, "I think the current climate particularly males generally regarded as being aggressors and not to be trusted... seems to be an automatic assumption... males who work in school in primary schools are potential paedophiles..." (Male, aged 59, 32 years, Principal). Conversely, another male participant had a very different experience and reported positive interactions with a female student who chose to confide in him. The male participant reported feeling confident of his efforts to create a safe environment for students to approach him, stating, "So for a year 10 girl to...tell me what's happened the night before with dad... felt a bit, I don't want to say special, but I felt like I did something in the past to encourage or make her feel safe in terms of coming to me" (Male, aged 25, 4 years, Year 10 Coordinator).

\section{Mixed Levels of Support and Training for Schools}

Mixed Levels of Trauma-Based and Trauma-Informed Practices for Handling DV Some participants reported varying levels of knowledge regarding trauma-informed practices and their implementation in school. For example, one participant stated, "We do a lot of trauma-informed practice, we have a lot of trauma based professional development for our staff" (Female, aged 47, 20 years, Principal). However, other participants mentioned not having any trauma-informed protocols at their school or there being an absence of knowledge regarding the concept trauma-informed practice. This was reflected by a participant who stated, "No I haven't really, no. I mean obviously I know what trauma is, but yeah, no, I know that trauma can come in all different forms, but no I don't have any practice in that, no" (Female, aged 39, 15 years, Principal).

Some participants mentioned the importance of creating a sense of safety for students exposed to DV. Using mindfulness practices and taking breaks during class were reported to help students regulate their emotions and focus on their work. One participant stated, "So we do a lot of work at the school and putting it part of our daily practices of doing things like mindfulness and trying to get them to be more in the present, and that will help them with their emotional regulation and hopefully being able to feel safe" (Female, aged 36, 9 years, Principal). However, there were also challenges to implementing support for students. Some participants mentioned that parental consent can be a barrier for students accessing individualised emotional support. In one instance, a school was reportedly unable to allow students to access mental health support via the school chaplain if the parents did not consent. The participant stated, "Parents have to give consent for the chaplain to be able to work with the student. If they haven't given consent well that's a barrier" (Female, aged 36, 15 years, Principal).

Differing Levels of Support for Schools to Deal with Parents and Families Participants' reported that their interactions with parents who report DV were often difficult because they lack training in this area. Participants indicated a sense of frustration with parents who they perceive as placing their own needs above those of their children. Participants were unsure whether their role was to only to support students or whether they had a duty to support the parents as well. Additionally, many participants felt that parents did not follow through with suggestions given by the school with one participant stating, "Although we said that we don't have a responsibility to the parents, they come and they throw themselves at our a [sic] mercy a lot of the time and so it's like, well, what's appropriate and - to deal with them?" (Female, aged 36, 12 years, Assistant Principal).

Some participants reported that, at times, parents show verbal aggression towards school staff, including parents with whom the school has previously had a positive relationship with. One participant stated, "I just think that because at the end of the day the parents or whoever, if they've disclosed of information, it can backfire on us, and you know having parents quite irate with us, or it can backfire on the children" (Female, aged 39, 15 years, Principal). Another explained that "Through my principalship, I've had some really great parents who are able to totally flip it and it's all your fault, and you just think what happened there...The best thing that I've learnt is about being reflective and actually finishing a meeting” (Female, aged 47, 20 years, Principal). Aggression shown by parents often made participants doubt their ability to handle conflict with parents. Therefore, participants stated the need for more training specific to managing parental aggression.

Mixed Levels of Resources and Training for School Staff to Support Students Participants reported needing training to appropriately respond to students exposed to DV. Participants reported that they needed training on how to communicate with empathy when a student discloses DV, and training on how to improve the learning environment for DV-exposed students. Some participants reported a lack of confidence in detecting situations of DV. Most participants reported having undergone training on topics related to DV but not specifically about DV, such as mandatory reporting training or trauma-related training. Participants reported that experience was the best way to be acquainted with how to 
support students who have been exposed to DV but also highlighted that it remains an uncomfortable and stressful experience. For example, one participant stated, "So I think always prepared from experience but...then something that I have never dealt with happens...but I am always nervous and I am always anxious" (Female, aged 36, 15 years, Principal). Participants also reported it being hard for the schools to provide individualised support to all students due to the lack of time and resources. One participant stated, "I think one of the biggest ones for me is the time factor" (Female, aged 50, 25 years, Year Level Coordinator).

\section{Levels of School Support from Government for DV Victims}

Funding for Schools Some participants reported that schools are under-resourced and lack financial resources, which then prevents school from being able to support students, parents and families affected by DV. One participant felt that while general funding for students with additional needs can be helpful, support for schools should be individualised. Specifically, participants felt that funding should be allocated based on the needs of the school instead of being determined based the number of student enrolments. Further, participants reported inconsistency in the levels of funding allocated to supporting schools manage social issues such as DV, making it difficult for schools to offer consistent intervention at a student and whole-school level. This was echoed by the following participant who reported: "Funding that's been helpful but it's unpredictable so is it likely to continue or is that going to change when the government changes and you just don't know" (Female, aged 36, 15 years, Principal).

Support from External Agencies Participants cited that community health services also have limited resources and therefore, are unable to support all students and families impacted by DV. Participants suggested that this lack of resources meant the responsibility to support students exposed to DV often fell back onto schools. For example, one participant stated, "The Education Department embraces a more community approach to schools...it's not matched really with resources...so in a school such as mine, which is an hour away from a major city or a major rural centre, you have to develop your own skill set... and respond to issues such as domestic violence" (Male, aged 60, 32 years, Principal).

Participants also discussed several challenges in dealing with external agencies, such as child protection services and community service organisations. Participants were at times dissatisfied with the outcomes for students as a result of involving external services and with the lack of ongoing support from external services for students exposed to DV. Additionally, participants reported a lack of communication with child protection services regarding their investigation into a DV case, with one participant stating, "But I know that when you start escalating things it, it can take quite some time to get response from people that, in the Government for example in Departments, they can be quite slow getting back to you" (Male, aged 59, 32 years, Principal). Participants further mentioned that this lack of communication with authorities limited their understanding of the students' issues and therefore impeded their ability to support the student involved. One participant stated, "I can't speak to certain people unless the investigation is finished and even though they are an amazing support and it's their job to investigate...it's communication - that timely transfer of information so that I am able to support the other children that may have heard what's happened" (Female, aged 57, 30 years, Principal).

\section{Role of Wellbeing Officers}

Reliance on School Wellbeing Officers for Support Participants reported relying on the wellbeing officers in their school for information regarding student mental health concerns. The wellbeing staff at participants' schools comprised of wellbeing coordinators, counsellors, school chaplains, as well as other staff members who have experience in a wellbeing role. Participants reported that wellbeing staff are helpful in terms of providing support to both the participants and to other members of the school community. One participant stated, "We've got a very good wellbeing team so they kind of - they're the first port of call so that if a lot of stuff comes up, often teachers and myself would have recognized maybe there might be something a little bit off with the student" (Female, aged 36, 12 years, Assistant Principal). Another participant expressed a similar sentiment, stating, "We've been very lucky to employ very skilled practitioners-I've got a very, very skilled chaplain and I've got a wellbeing coordinator who is an education support staff member" (Male, aged 60, 32 years, Principal).

School Wellbeing Departments' Knowledge of Mental Health Support Wellbeing officers were perceived by participants as being more capable of providing mental health support to students than teachers. Wellbeing officers were cited to have better knowledge of mandatory reporting legislation for child abuse and neglect, and more practical knowledge regarding how to help students exposed to DV. For example, one participant stated, "And if it gets too much, you handball them off to someone above you, like the wellbeing team or even AP's and that kind of thing. So I do feel like I'm very limited to what I can do" (Male, aged 25, 4 years, Year Level Coordinator). Wellbeing officers were also reported by participants to be a valuable source of support and debriefing for other members of staff. Participants cited a hierarchy in 
which they referred students onto school wellbeing officers for situations they felt unable to handle. However, participants also voiced that meetings between students and wellbeing officers often impacted on students' academic work. Participants felt that when students miss classes, this affected classroom teachers and lowered students' academic performance. Participants acknowledged that students need help from wellbeing officers but were not sure how to balance students' academic needs with their wellbeing needs, with one participant stating, "Well, if you're going out to see wellbeing and you're not in class, well, then, that's having an impact on me as a teacher and so how much - where's that line, that maybe needs to be crossed" (Female, aged 36, 12 years, Assistant Principal).

\section{Trauma Training and the Impact on Staff}

Participants reported that some staff members had been personally victimised by DV and therefore, were mindful of the potential impacts of trauma-training on staff wellbeing. One school leader mentioned the use of videos when providing trauma-based training for staff caused distress to some of the school staff. For example, one participant stated, "I didn't realise that until we understand vicarious trauma - these activities were most likely the worst thing in I could have done last year because it traumatised half the staff" (Female, aged 57, 30 years, Executive Principal).

Participants suggested that face-to-face training impacted staff differently; for example, school staff with prior wellbeing training felt face-to-face training refreshed their DV response skills, while others developed increased empathy for students exposed to DV. One participant indicated that school staff are not satisfied with online training and recommended that training should be done face-to-face by people who have had training and experience in dealing with similar situations as school staff. Participants indicated that the large workload of staff was a barrier to attending online training and recommended that training should be either face-to-face or non-compulsory. This sentiment was echoed by one participant to who stated, "Staff are over online training. You need face to face - people that are trained and prepared and have dealt with this" (Female, aged 57, 30 years, Executive Principal). Another participant stated "Some classroom teachers they're just so insanely busy that I do think our workload is so massive that maybe opt-in stuff [training] but not forced" (Female, aged 38, 14 years, Year Level Coordinator).

\section{Discussion}

In response to a gap in the literature, the current study explored the experiences of school leaders regarding students exposed to DV, the perceived barriers in supporting these students, and school leaders' recommendations to improve educational services for DV-exposed students and families. School leaders are arguably in a much more challenging situation when compared to other school staff in regard to student wellbeing concerns and responsibility over students, parents, teachers and school wellbeing staff. The Equality Institute (2018) indicated schools play a significant role in facilitating safety for children who experience DV at home. Further, schools are identified by children who have experienced DV as protective and a retreat from the challenges of home (Equality Institute, 2018; O'Brien et al., 2013). School leaders felt positive about their role supporting students exposed to DV in terms of providing a safe and secure school setting and facilitating students' access to school-based support. Nevertheless, providing a safe and supportive school environment for these students is reportedly complex and multifaceted.

The results suggest education staff are regularly balancing the educational and social-emotional needs of these students with the needs of the broader classroom and the capacity of teaching staff. Children exposed to DV experience impaired educational attainment, limited behavioural control, emotional dysregulation, poorer self-esteem and increased secrecy around family circumstances (Lloyd, 2018). Principals in the current study highlighted these issues and the emotional toll of students' disclosure of DV for school staff. When supporting families in the context of DV, school leaders described difficult conversations with children and families about home-life. Families reportedly become defensive and worried about how they are perceived by the school. Consistent with previous research, the current study found school leaders navigate conversations with irate or aggressive parents and manage reduced parental school engagement and poor student attendance (Schnurr \& Lohman, 2013), all of which require a great amount of individualised support for the student.

Findings relating to DV-specific school protocols highlighted diverse views about the extent to which schools should intervene and how prepared staff are to respond to DV-exposed students. Cameron (2016) found childcare staff believed unrealistic expectations were placed on them as educators to respond to complex social issues such as DV. Consistent with the current study, educators often lack confidence and knowledge to manage DV (Lloyd, 2018). Further, educators have consistently emphasised that they require clarification of their role, training and practice frameworks to inform their response to DV (Cameron, 2016; Lloyd, 
2018). Evidently, without appropriate levels of training and support, school leaders report feeling ill-equipped to manage DV, and thus, may be less likely to intervene, placing children at increased risk (Davies \& Ward, 2012; Lloyd, 2018).

The current study found school leaders preferred an 'opt in' approach to DV training for staff. It was suggested that training should take into account the high workload and potential distress caused to staff when attending DV training. However, the benefits of training identified by participants, such as increased skills and greater empathy for children and families, provide a strong rational for why adequate and accessible training should be a priority. O'Brien and colleagues (2013) suggested that DV education and training should consider competing demands and limited time of staff. The current study found principals had a preference for in-person training. Online learning was identified as potentially triggering. Face-to-face training may facilitate more supportive and engaging learning experiences. The delivery of face-to-face training was also recommended at the Royal Commission into Family Violence in Victoria to promote active engagement in the training material (Neave et al., 2016). It was recommended that training address the gendered nature of DV and increase the capacity of male school leaders to respond to disclosures in a safe way. School leaders believed upskilling male staff is an important component of the school DV-response and students require access to supportive female staff if approaching a male school leader is too difficult for students. On the other hand, some students may respond better to male staff rather than female staff if their exposure to DV has taught them to disrespect females. This research did not intend to explore the issue of gender, sexuality and school leaders' experiences responding to students exposed to DV, however, it is recommended that research continue in this area in future.

The current study suggests to provide effective responses to DV, organisations, including schools, cannot act in isolation (Byrne \& Taylor, 2007; Neave et al., 2016). Consistent with previous research, collaboration between education services, the family violence sector and other community health and welfare organisations (such as Child Protection) is essential to promote holistic, community care of students experiencing DV (Cameron, 2016). This study highlighted the challenge of navigating confidentiality. Staff perceived that services are unable to share information that could improve their response and support for students experiencing DV.

School leaders were generally conscious and sensitive to staff members' personal experiences of DV and relied heavily on school wellbeing staff to offer debriefing, education and support to staff responding to student disclosures of DV. However, the current study points to a lack of formalised training and response frameworks across the education system, and as such, staff support was inconstant across schools. The Victorian State Government (2019) recently commenced efforts to roll out a standardised, school-based approach to family violence focusing on gender equality, support for victims, accountability for perpetrators and prevention through systemic measures. Although ongoing evaluation is required to determine the effectiveness of these measures, this new approach may assist schools in response to DV, with school leaders in the current study calling for more information, training and resources on the matter.

\section{Implications of the Study}

Consistent with a review by the Equality Institute (2018), the current study suggests improvement of school-based DV policies and protocols, community partnerships, sector-wide training and capacity building is needed, as well as ongoing research and evaluation to enhancing the preparedness of school leaders to understand and respond to DV. While education staff are not expected to be specialists in this area, the current study found school leaders and teachers would benefit from a deeper understanding of the nature and impact of DV on children and families. Training that is delivered to school staff should take into account the gendered nature of $\mathrm{DV}$, the specific learning needs of staff, and possible triggers for staff who have experienced DV themselves. Some school leaders suggested DV training should be optional. However, research suggests that a comprehensive, wholeschool approach is likely to lead to more positive outcomes for staff and students. Further, school leaders may require specialist training given their responsibility over students and staff. In particular, training for school leaders should address identifying signs of DV, managing difficult conversations with hostile parents concerning DV, and implementation of school-wide responses to DV.

School leaders also reflected that it may not be necessary or realistic for all staff to attend DV training. Nevertheless, best practice points to whole-school approaches which utilise a trauma-informed lens (Equality Institute, 2018). Multitiered trauma interventions could be a method for providing training to all school staff, while also acknowledging that school leaders and wellbeing staff require more specialist training and skills compared to teachers. Within a multitiered model of training, school leaders and wellbeing staff are trained to intervene with students and families impacted by DV. Further, they receive training regarding how to support and coach teachers exposed to these students. Teachers on the other hand are given professional development on noticing and referring students who have disclosed DV (Berger, 2019).

A multi-tiered approach to training for staff could be a viable option to improve the sustainability and uptake of 
professional development for staff in schools. An example of one such intervention is the Healthy Environments and Response to Trauma in Schools (HEARTS) program (Dorado et al., 2016). This program aims to improve training for school staff and support for students exposed to trauma through different levels of training and support at school. This approach could be a viable pathway to improve the sustainability and uptake of professional development for school staff. A program evaluation of the HEARTS model found significant improvements in 1) staff knowledge of trauma and its impact on children, 2) staff understanding of how to support children exposed to trauma, and 3) staff knowledge about trauma-informed practices (Dorado et al., 2016). It is worth noting that the HEARTS program only has one publically available evaluation and trauma-informed program evaluations often do not investigate outcomes for teachers and school leaders (Berger, 2019). There is currently limited emphasis beyond this research on the impact of DV exposure and training among school leaders.

It is also important to note that the current COVID-19 pandemic has increased the need for school resources and policies to manage the risk of DV for families and children (Peterman et al., 2020). COVID-19 required many schools and healthcare services to operate online (Berger \& Reupert, 2020; Huber \& Helm, 2020), meaning that online training for school staff concerning DV may be necessary. However, the identification of DV within families also changed, with many students and parents being observed in their homes via online learning platforms rather than face-to-face. Online learning and observations of students at home may result in teachers suspecting DV exposure for more students, highlighting the timely importance of this research and DV training and support for school staff.

\section{Limitations}

While the sample size of 15 is common for qualitative research, future research could replicate the current study using a larger sample size and points of data saturation (Fusch \& Ness, 2015). Further, broader sampling of school leaders in other states or countries may enhance generalisability of the results. Another limitation is the lack of gender diversity in the sample. Participants identified as either female or male and the sample did not include perspectives from principals who identified as LGBTQI. Furthermore, two out of the three male participants were in a principal position, meaning a lack of diversity in male school leaders in roles other than the role of principal. The current study suggests that the gender of school leaders may influence the relationship and interactions with students and families regarding DV. Therefore, future studies may choose to specifically target male, cisgender, heterosexual or LGBTQI school leaders and aim for larger sample sizes.
As this study sample involved school leaders of various roles and from different school settings (e.g., primary schools, secondary schools, special education schools), the sample was not homogenous. This study also did not collect details on participant's identified race at the beginning of the interview. Race is another important consideration for future research to understand how a teacher's race may shape their perspectives and experiences with DV-exposed students. Future research may benefit from replicating this study to explore each of the identified themes in more detail and target groups by position in school, race, gender, sexuality and type of school setting.

\section{Conclusion}

This study explored the experiences, barriers and recommendation of school leaders regarding students' DV experiences. Results indicated that systemic processes, such as the implementation of schoolwide policy and procedure, could be improved to increase the capacity of schools to respond to DV. However, external support and collaborative community relationships were also highlighted as essential to equip school staff to support students and families impacted by DV. The barriers that school leaders and staff experience included limited DV-specific training, secrecy and confidentiality surrounding DV, difficulty establishing safe and trusting relationships with parents, and challenges obtaining external support for DV victims. Overall, school leaders indicated that schools and school staff are committed to support students exposed to DV and recommended that DV training and protocols are needed in schools to improve the learning environment for DVexposed students.

\section{Declarations}

Conflict of Interest The authors have no conflicts of interest to declare with respect to this research.

\section{References}

Alisic, E. (2012). Teachers' perspectives on providing support to children after trauma: A qualitative study. School Psychology Quarterly, 27(1), 51-59. https://doi.org/10.1037/a0028590

Australian Government. (2011). National plan to reduce violence against women and their children 2010-2022. Author.

Australian Institute of Family Studies. (2020). Mandatory reporting of child abuse and neglect, CFCA Resource Sheet-June 2020. https://aifs.gov.au/cfca/publications/mandatory-repor ting-child-abuse-and-neglect\#: : text=How\%20does\%20man datory\%20reporting\%20legislation, children $\% 20$ aged $\% 20$ und er\%2016\%20years. 
Benoliel, P. (2017). Managing senior management team boundaries and school improvement: An investigation of the school leader role. International Journal of Leadership in Education, 20(1), 57-86. https://doi.org/10.1080/13603124.2015.1053536

Berger, E. (2019). Multi-tiered approaches to trauma-informed care in schools: A systematic review. School Mental Health, 1-15. https:// doi.org/10.1007/s12310-019-09326-0

Berger, E., \& Reupert, A. (2020). The COVID-19 pandemic in Australia: Lessons learnt. Psychological Trauma: Theory, Research, Practice, and Policy, 12(5), 494-496. https://doi.org/10.1037/ tra0000722

Boxall, H., Dowling, C., \& Morgan, A. (2020). Female perpetrated domestic violence: Prevalence of self-defensive and retaliatory violence. Trends and Issues in Crime and Criminal Justice, 584, 1.

Braun, V., \& Clarke, V. (2006). Using thematic analysis in psychology. Qualitative Research in Psychology, 3(2), 77-101. https://doi.org/ 10.1191/1478088706qp063oa

Buckley, H., Holt, S., \& Whelan, S. (2007). Listen to me! Children's experiences of domestic violence. Child Abuse Review, 16(5), 296-310. https://doi.org/10.1002/car.995

Byrne, D., \& Taylor, B. J. (2007). Children at risk of domestic violence and their educational attainment: Perspectives of education welfare officers, social workers and teachers. Child Care in Practice, 13(3), 185-201. https://doi.org/10.1080/1357527070 1353465

Cameron, P. (2016). Expanding early interventions in family violence in Victoria. Domestic Violence Victoria.

Campo, M., \& Tayton, S. (2015). Intimate partner violence in lesbian, gay, bisexual, trans, intersex and queer communities. Australian Institute of Family Studies, 1(1), 1-7.

Davies, C., \& Ward, H. (2012). Safeguarding Children Across Services: Overview of Key Messages from 15 English Research Studies. Jessica Kingsley Publishers. https://doi.org/10.1037/e516542013-192

Davies, S., \& Berger, E. (2019). Teachers' experiences in responding to students' exposure to domestic violence. Australian Journal of Teacher Education, 44(11), 96-109. https://doi.org/10.14221/ ajte.2019v44.n11.6

Department of Families, Housing, Community Services and Indigenous Affairs \& Council of Australian Governments. (2011). National plan to reduce violence against women and their children. Department of Families, Housing, Community Services and Indigenous Affairs.

Dorado, J. S., Martinez, M., McArthur, L. E., \& Leibovitz, T. (2016). Healthy Environments and Response to Trauma in Schools (HEARTS): A whole-school, multi-level, prevention and intervention program for creating trauma-informed, safe and supportive schools. School Mental Health, 8(1), 163-176. https://doi.org/10. 1007/s12310-016-9177-0

Drysdale, L., \& Gurr, D. (2011). Theory and practice of successful school leadership in Australia. School Leadership \& Management, 31(4), 355-368. https://doi.org/10.1080/13632434.2011.606273

Equality Institute. (2018). Family Violence and Education: Review of the Evidence on Impacts of Violence and Responding to Violence in the Education Sector. https://www.equalityinstitute.org/ media/pages/resources/family-violence-and-education-reviewof-the-evidence-on-impacts-of-violence-and-responding-to-viole nce-in-the-education-sector/1379954212-1595397622/det_lowres-double-pages.pdf

Falkiner, M., Thomson, D., \& Day, A. (2017). Teachers' understanding and practice of mandatory reporting of child maltreatment. Children Australia, 42(1), 38-48. https://doi.org/10.1017/cha.2016.53

Fusch, P. I., \& Ness, L. R. (2015). Are We There Yet? Data Saturation in Qualitative Research. The Qualitative Report, 20(9), 1408-1416. https://www.proquest.com/scholarly-journals/arewe-there-yet-data-saturation-qualitative/docview/1721368991/ se-2? accountid $=12528$.
Garcia-Moreno, C., Jansen, H., Ellsberg, M., Heise, L., \& Watts, C. H. (2006). Prevalence of intimate partner violence: Findings from the WHO multi-country study on women's health and domestic violence. The Lancet (British Edition), 368(9543), 1260-1269. https://doi.org/10.1016/S0140-6736(06)69523-8

Howard, S. K., Curwood, J. S., \& McGraw, K. (2018). Leaders fostering teachers' learning environments for technology integration. In Second Handbook of Information Technology in Primary and Secondary Education (pp. 515-533).

Huber, S. G., \& Helm, C. (2020). COVID-19 and schooling: Evaluation, assessment and accountability in times of crises-reacting quickly to explore key issues for policy, practice and research with the school barometer. Educational Assessment, Evaluation and Accountability, 32, 237-270. https://doi.org/10.1007/ s11092-020-09322-y

Iacona, J., \& Johnson, S. (2018). Neurobiology of trauma and mindfulness for children. Journal of Trauma Nursing: The Official Journal of the Society of Trauma Nurses, 25(3), 187-191. https://doi.org/ 10.1097/JTN.0000000000000365

Kuskoff, E., \& Parsell, C. (2019). Preventing domestic violence by changing australian gender relations: Issues and considerations. Australian Social Work, 73(2), 227-235. https://doi.org/10.1080/ 0312407x.2019.1641529

Levendosky, A. A., \& Graham-Bermann, S. A. (2001). Parenting in battered women: The effects of domestic violence on women and their children. Journal of Family Violence, 16(2), 171-192. https://doi.org/10.1023/A:1011111003373

Levendosky, A. A., Leahy, K. L., Bogat, G. A., Davidson, W. S., \& von Eye, A. (2006). Domestic violence, maternal parenting, maternal mental health, and infant externalizing behavior. Journal of Family Psychiatry, 20(4), 544. https://doi.org/10.1037/0893-3200. 20.4.544

Lloyd, M. (2018). Domestic violence and education: Examining the impact of domestic violence on young children, children, and young people and the potential role of schools. Frontiers in Psychology, 9(2094), 1-11. https://doi.org/10.3389/fpsyg.2018.02094

Mathews, B., Ju Lee, X., \& Norman, R. E. (2016). Impact of a new mandatory reporting law on reporting and identification of child sexual abuse: A seven-year time trend analysis. Child Abuse \& Neglect, 56, 62-79. https://doi.org/10.1016/j.chiabu.2016.04.009

McKee, B. E., \& Dillenburger, K. (2009). Child abuse and neglect: Training needs of student teachers. International Journal of Educational Research, 48(5), 320-330. https://doi.org/10.1016/j.ijer. 2010.03 .002

Moore, B. (2009). Emotional intelligence for school administrators: A priority for school reform? American Secondary Education, 37(3), 20-28.

Neave, M., Faulkner, P., \& Nicholson, T. (2016). Royal Commission into Family Violence Summary and recommendations. State of Victoria. Retrieved from: https://www.parliament.vic.gov.au/file_ uploads/1a_RFV_112ppA4_SummaryRecommendations.WEB_ DXQyLhqv.pdf

O’Brien, K. L., Cohen, L., Pooley, J. A., \& Taylor, M. F. (2013). Lifting the domestic violence cloak of silence: Resilient Australian women's reflected memories of their childhood experiences of witnessing domestic violence. Journal of Family Violence, 28(1), 95-108.

Peterman, A., Potts, A., O’Donnell, M., \& Thompson, K., Shah, N., Oertelt-Prigione, S., \& van Gelder, N. (2020). Pandemics and Violence Against Women and Children. Center for Global Development Working Paper 528. Retrieved from https://www.cgdev.org/ publication/pandemics-and-violence-against-women-and-children

Pitts, M., Smith, A., Mitchell, A., \& Patel, S. (2006). Private lives: A report on the health and wellbeing of GLBTI Australians. Australian Research Centre in Sex, Health \& Society. https://apo.org.au/ sites/default/files/resource-files/2006-02/apo-nid4050.pdf. 
Reuter, T. R., Newcomb, M. E., Whitton, S. W., \& Mustanski, B. (2017). Intimate partner violence victimization in LGBT young adults: Demographic differences and associations with health behaviors. Psychology of Violence, 7(1), 101-109. https://doi. org/10.1037/vio0000031

Savin-Baden, M., \& Howell Major, C. (2013). Qualitative research: The essential guide to theory and practice. Routledge.

Schnurr, M. P., \& Lohman, B. J. (2013). Longitudinal impact of toddlers' exposure to domestic violence. Journal of Aggression, Maltreatment \& Trauma, 22(9), 1015-1031. https://doi.org/10.1080/ 10926771.2013.834019

Smith, M. C. (2010). Early childhood educators: Perspectives on maltreatment and mandated reporting. Children and Youth Services Review, 32, 20-27. https://doi.org/10.1016/j.childyouth.2009.06. 011

State Government of Victoria, Australia (2019). Child Protection Reporting Obligations. Retrieved from https://www.education. vic.gov.au/school/principals/spag/safety/Pages/childprotectobl igation.aspx

United Nations Population Fund. (2000). The state of world population: Lives together, worlds apart - Men and women in a time of change. United Nations. https://www.unfpa.org/publications/ state-world-population-2000.

Vaughan-Jensen, J., Smith, D. M., Blake, J. J., Keith, V. M., \& Willson, V. K. (2020). Breaking the cycle of child maltreatment and intimate partner violence: The effects of student gender and caring relationships with teachers. Journal of Aggression, Maltreatment \& Trauma, 29(2), 223-241. https://doi.org/10.1080/10926771. 2018.1522407

Victorian State Government. (2016). Protect: Identifying and responding to all forms of abuse in Victorian schools. Department of education and training. Victorian State Government.

Victorian State Government. (2019). Teach respectful relationships. https://www.education.vic.gov.au/school/teachers/teachingre sources/discipline/capabilities/personal/Pages/respectfulrel.aspx\# link23

Whitaker, R. C., Orzol, S. M., \& Kahn, R. S. (2006). Maternal mental health, substance use, and domestic violence in the year after delivery and subsequent behavior problems in children at age 3 years. Archives of General Psychiatry, 63(5), 551-560.

Yates, S. (2020). Gender, context and constraint: Framing family violence in Victoria. Women's Studies International Forum, 78, 102321. https://doi.org/10.1016/j.wsif.2019.102321

Publisher's Note Springer Nature remains neutral with regard to jurisdictional claims in published maps and institutional affiliations. 